\title{
Thorn-Shape Waveform and Double-Strike Phenomenon Seen in the Impact Force of Soft Materials
}

\author{
Yukio Fujimoto, Chen Liu, Yoshikazu Tanaka, Eiji Shintaku, Toshiki Nakanishi \\ Department of Transportation and Environmental Engineering, Graduate School of Engineering, Hiroshima \\ University, Hiroshima, Japan \\ Email: fujimoto@naoe.hiroshima-u.ac.jp
}

Received 25 March 2015; accepted 21 April 2015; published 23 April 2015

Copyright (C) 2015 by authors and Scientific Research Publishing Inc.

This work is licensed under the Creative Commons Attribution International License (CC BY). http://creativecommons.org/licenses/by/4.0/

(c) (i) Open Access

\begin{abstract}
In the impact tests of soft materials, we sometimes observe a thorn shape in the rising segment of the impact force waveform. However, the reason for the occurrence of the thorn shape has not been made clear. In this study, thorn-shape waveforms of several soft materials are measured using compact drop test equipment under the condition of a flat frontal impact. A flat frontal impact is the condition where a drop hammer with a flat bottom surface strikes a plate-like soft material in the normal direction. Synchronized impact forces are measured using two sensors installed on both the drop hammer side and the floor side. The examined soft materials are a sponge sheet, sponge rubber sheet, gel sheet, rubber sheet, flat oil clay, low-rebound urethane foam, cork sheet, sliced ham, pork ham steak, and pork. Based on the test results, the features of the thorn-shape waveforms are discussed from a bird's-eye view. Furthermore, the occurrence mechanism of the thorn-shape waveforms is discussed from the viewpoint of viscosity discontinuity and the doublestrike phenomenon.
\end{abstract}

\section{Keywords}

Impact Force, Thorn-Shape Waveform, Soft Material, Drop Hammer, Viscosity Discontinuity, Strain Rate Dependence, Double-Strike Phenomenon

\section{Introduction}

In impact tests of soft materials such as rubber, clay, or soft plastic, we sometimes observe a thorn shape in the rising segment of the impulsive force waveform. Several thorn-shape waveforms can be seen in the impact test

How to cite this paper: Fujimoto, Y., Liu, C., Tanaka, Y., Shintaku, E. and Nakanishi, T. (2015) Thorn-Shape Waveform and Double-Strike Phenomenon Seen in the Impact Force of Soft Materials. World Journal of Mechanics, 5, 59-69.

http://dx.doi.org/10.4236/wjm.2015.54007 
results of previous research studies [1]-[3]. However, the physical meaning of the thorn shape has not been discussed much.

Recently, we have developed an impact force sensor that can measure a high-speed impact force waveform accurately [4] [5], and we have performed impact tests on several soft materials using the sensor. While repeating the experiments, the authors realized that the thorn-shape waveform can be clearly measured under the condition of a flat frontal impact. Also, the authors pointed out the possibility that the thorn-shape waveform is related to the strain rate dependence of the material and the double-strike phenomenon [6].

In this study, free drop impact tests are performed on several soft materials using compact drop test equipment. The thorn-shape waveforms are observed from a bird's-eye view. Further, the occurrence mechanism of the thorn-shape waveform is discussed from the viewpoint of viscosity discontinuity and the double-strike phenomenon.

\section{Thorn Shape and Double-Strike Phenomenon Seen in the Impact Force Waveform of Soft Materials}

Figure 1(a) shows a soft material subjected to a flat frontal impact. The impact object is a free drop hammer. Figure 1(b) shows typical thorn-shape waveforms of the impact force. The features of the thorn-shape waveform are a steep slope on the rising segment (which is $\theta$ ) and a thorn-shape waveform similar to the yield point of low-carbon steel. After that, at a certain point, it becomes a gentler slope (which is $\alpha$ ) and generates a second mountain-shape waveform. The rising segment of the thorn is usually close to a straight line.

Figure 1(c) summarizes the occurrence mechanism of thorn-shape waveforms obtained by the following experimental observation. The impact force waveform of soft materials is the mixture of two types of waveforms. The first (thorn-shape) waveform shows a large viscosity resistance (red curve), and the second (mountain-shape) waveform shows a small viscosity resistance (dashed black curve). This resistance change occurs suddenly at the thorn peak; that is, a viscosity discontinuity (viscosity transient) is induced at the thorn peak. The compressive stress at the thorn peak is the trigger of the softening. The soft material surface is struck twice by the relative movement of collision objects. The reason why the slope $\alpha$ becomes smaller than the initial slope $\theta$ is because a viscosity discontinuity is induced by the first strike.

Figure 2 shows three types of thorn-shape waveforms measured in the following experiments:

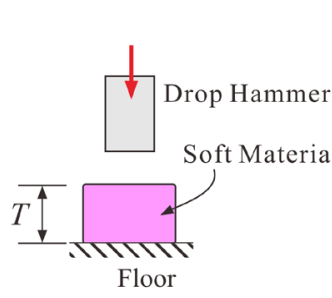

(a)

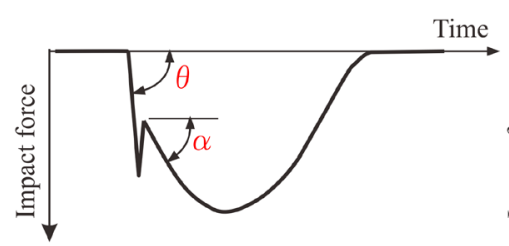

(b)

(Q): Thorn-shape waveform

(R): Mountain shape waveform

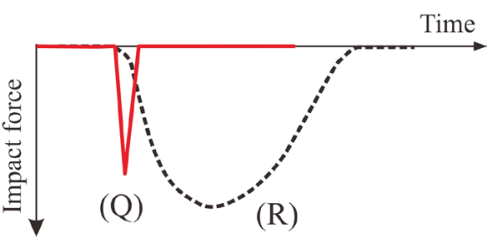

(c)

Figure 1. Impact force waveform of soft material. (a) Flat frontal impact by free drop hammer and plate-like soft material; (b) Typical impact force waveform of soft material including thorn; (c) Two types of waveforms contained in the impact force waveform of soft material.

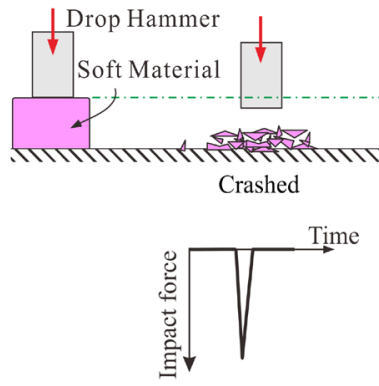

Type I

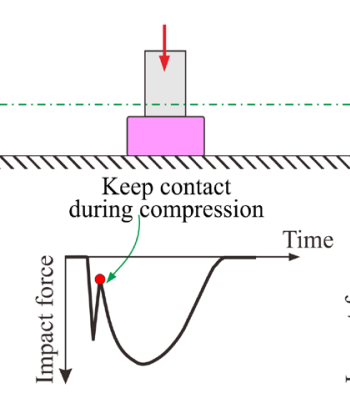

Type II

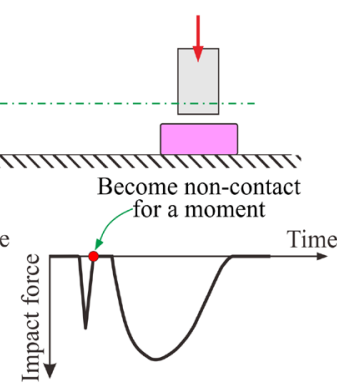

Type III

Figure 2. Three types of thorn-shape waveforms observed in the impact force of soft material. 
Type I: In the case where the soft material is crushed into pieces by the first impact, only a thorn-shape waveform appears, and the second mountain-shape waveform does not appear.

Type II: This type of waveform appears in most of the soft materials. The slope $\theta$ and thorn peak are influenced by the material, impact velocity, material thickness, etc. In this waveform, a certain compressive force still remains after the first impact. This means that the hammer bottom and soft material surface remain in contact during unloading.

Type III: This type of waveform is observed in the thick soft material. The hammer bottom and the soft material surface have a non-contact condition for a moment after the first impact.

\section{Condition of Flat Frontal Impact and Compact Drop Test Equipment}

Through a number of experiments, we found that the thorn-shape waveform can be measured clearly under the condition of a flat frontal impact. In Figure 3, the impact object is a solid material with a flat contact surface. The soft material that receives the impact force is a plate-like material with a uniform thickness without surface irregularities. The impact object strikes the soft material in the normal direction. The thorn-shape waveform can be steadily measured in the above condition. When the bottom surface of the impact object is curved (Figure 3(b)), when the impact object is tilted (Figure 3(c)), or when the upper surface of the soft material is curved (Figure 3(d)), the thorn becomes unclear. Here, "unclear" does not mean that the thorn will not occur; it is unclear in that the contact area gradually expands from the local area, and the phenomenon is diluted.

Figure 4 shows the free drop test equipment used in this study. The device is braced by vertical columns on the left and right of the bottom plate. The top of the side column is connected with a metal plate. The drop hammer is made of an aluminum circular disk plate and a stainless cylinder (mass of $1.9 \mathrm{~kg}$ ). The contact surface of the hammer bottom is a disk plate with a diameter of $D=60 \mathrm{~mm}$. The upper sensor is an orbicular pad sensor and is attached near the drop hammer bottom. The lower sensor is a pad sensor of $100 \mathrm{~mm}$ square and is attached to the frame bottom. Both sensors are made from piezoelectric film. A high-speed voltage recorder with a sampling rate of $20-200 \mathrm{kHz}$ is used for recording the sensor output. The output of the upper sensor is modified by $8 \%$ to account for the mass of the disk plate. Based on rigorous tests using a servo-controlled testing machine and a high-rigidity load cell, it was confirmed that both sensors can measure the impact force very accurately when the contact time is in the range between $0.1 \mathrm{msec}$ and $2 \mathrm{sec}$. The drop height $h$ is the height from

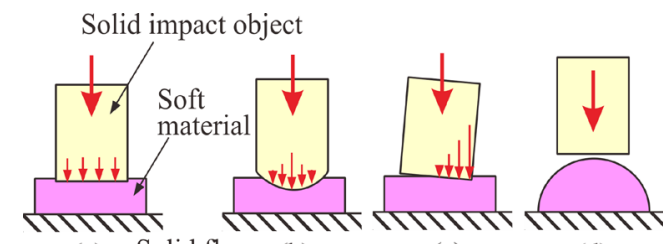

(a) Solid floor (b)

(c)

(d)

Figure 3. (a) Flat frontal impact condition that the thorn appears clearly. (b), (c), (d) are the conditions where the thorn becomes indistinct.
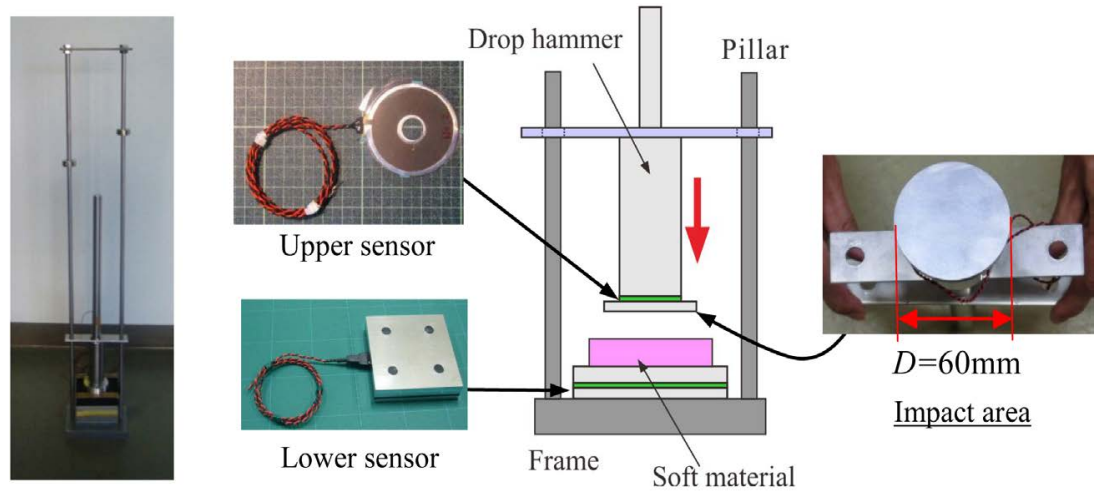

Figure 4. Drop test equipment and the upper and lower sensors used in the device. 
the upper surface of the soft material to the bottom surface of the drop hammer. The impact velocity is $V_{0}=1.4$ $\mathrm{m} / \mathrm{sec}$ when $h=0.1 \mathrm{~m}, V_{0}=1.98 \mathrm{~m} / \mathrm{sec}$ when $h=0.2 \mathrm{~m}$ and $V_{0}=2.73 \mathrm{~m} / \mathrm{sec}$ when $h=0.38 \mathrm{~m}$.

Figure 5 shows the importance of the flat frontal impact. Figure 5(a) shows woodblocks (triangular prism, semicircular column, and hemisphere) that are fixed to the bottom of the free drop hammer. The soft material is a nitrile rubber plate (NBR) with a $30 \mathrm{~mm}$ thickness. The drop height is $h=380 \mathrm{~mm}$. Figure 5(b) shows the measured impact force waveforms. The waveform under the flat frontal impact condition (without a wood block) is also shown in the figure. The thorn-shape waveform appears clearly under the flat frontal impact. However, when the block corner or a curved surface locally strikes the rubber, the thorn-shape waveform is not obvious.

\section{Experimental Results}

\subsection{Impact Force of Agar Jelly under Flat Frontal Impact (Type I Waveform)}

Figure 6 shows two examples of impact force waveforms for an agar jelly with a $30 \mathrm{~mm}$ thickness. The height of the drop hammer is $h=380 \mathrm{~mm}$. The initial strain rate at the collision is $\dot{\varepsilon}_{0}=V_{0} / T=91 \mathrm{sec}^{-1}$, where $V_{0}$ is the hammer speed at the collision, and $T$ is the soft material thickness. When the drop hammer collides, the agar jelly is crushed and scattered as juice. Therefore, the thorn-shape waveform (Type I waveform) appears only in the upper sensor output. The thorn is close to an isosceles triangle, and its contact time is short at $0.5 \mathrm{msec}$. The impulse of the thorn part is quite small compared with the total momentum of the drop hammer (5.19 N.sec). The impact force seen in the lower sensor output is delayed. This may be caused by the crush effect. Because the jelly was crushed at the thorn peak, the impact force is reduced to zero. About $15 \mathrm{msec}$ later, the hammer bottom strikes the lower sensor strongly without losing much of its falling velocity.

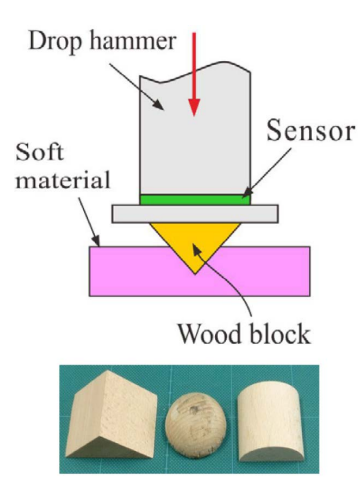

(a)

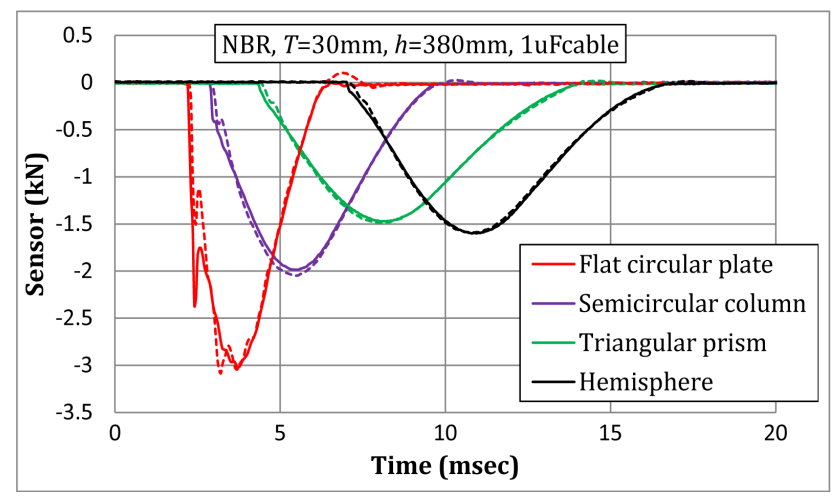

(b)

Figure 5. Influence of contact shape on impact force waveform. (a) Wood blocks attached to the drop hammer bottom; (b) Comparison of the waveform of the flat frontal impact and those of other impact conditions.

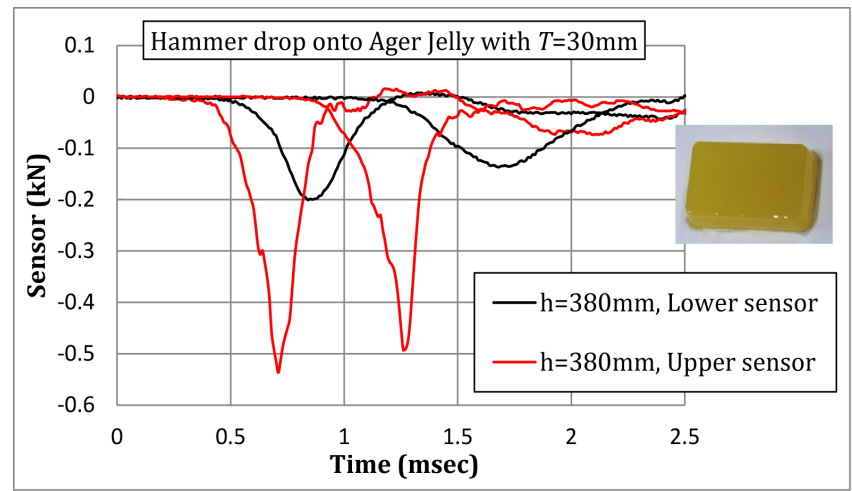

Figure 6. Impact force waveform induced by the hammer drop onto agar jelly. 


\subsection{Impact Force of Konjac under Flat Frontal Impact (Type III Waveform)}

Figure 7 shows the impact force waveforms and an enlarged view of a konjac plate with a $40 \mathrm{~mm}$ thickness. The drop height is $h=100 \mathrm{~mm}\left(\dot{\varepsilon}_{0}=V_{0} / T=35 \mathrm{sec}^{-1}\right)$ and $380 \mathrm{~mm}\left(\dot{\varepsilon}_{0}=68 \mathrm{sec}^{-1}\right)$. The konjac plate is compressed greatly by the impact but not crushed. In the figure, the time interval between the thorn-shape waveform and the succeeding mountain-shape waveform is wide, and the double-strike phenomenon is obvious (Type III waveform). In the magnified figure, the thorn is close to an isosceles triangle, and its contact time is short at 0.8 msec. Also, a small thorn can be seen in the lower sensor output. The impulse of the thorn part is quite small compared with the succeeding mountain-shape waveform. This means that most of the falling velocity is lost at the second mountain-shape waveform. Usually, the thorn shapes of the upper and lower sensor outputs are close when the material thickness is smaller.

\subsection{Impact Force of Gel Sheet under Flat Frontal Impact (Type II Waveform)}

Figure 8(a) shows the impact force waveforms of a gel sheet (product name: $\alpha$ gel) with a $20 \mathrm{~mm}$ thickness. Figure 8(b) is an enlarged view of the thorn part. The drop height is $h=380 \mathrm{~mm}\left(\dot{\varepsilon}_{0}=V_{0} / T=136 \mathrm{sec}^{-1}\right)$. The thorn-shape waveform can be seen clearly in both the upper and lower sensor outputs (Type II waveform). In the enlarged view, we can observe that the slope $\theta$ of the rising segment is larger than the slope $\alpha$ of the segment past the thorn. The impulse of the thorn part is quite small compared with the succeeding mountain-shape waveform.

\subsection{Impact Force of Nitrile Rubber (NBR) Plate under Flat Frontal Impact (Type II Waveform)}

Figure 9(a) is the impact force of an NBR plate with $T=30 \mathrm{~mm}$ (rubber hardness of 50). The drop height is

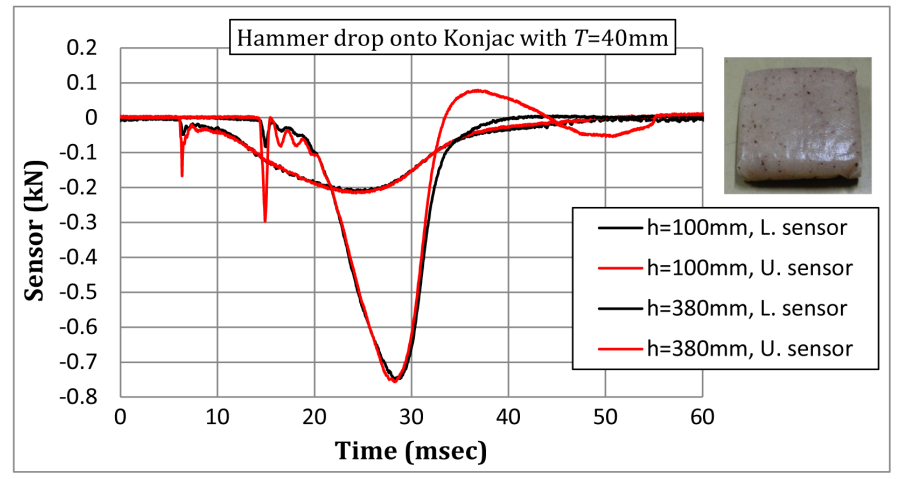

(a)

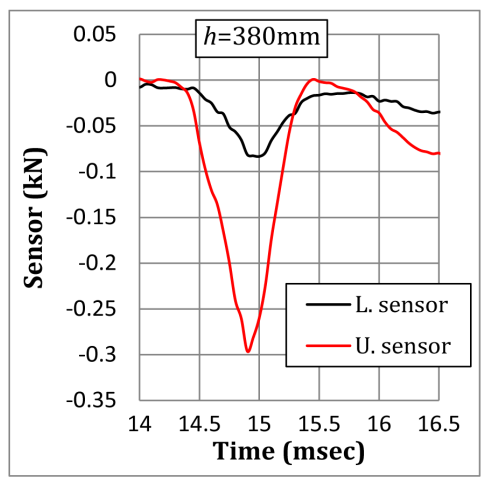

(b)

Figure 7. (a) Impact force waveforms induced by hammer drop onto konjac with a $40 \mathrm{~mm}$ thickness; (b) Enlarged figure of thorn part for the case of $h=380 \mathrm{~mm}$.

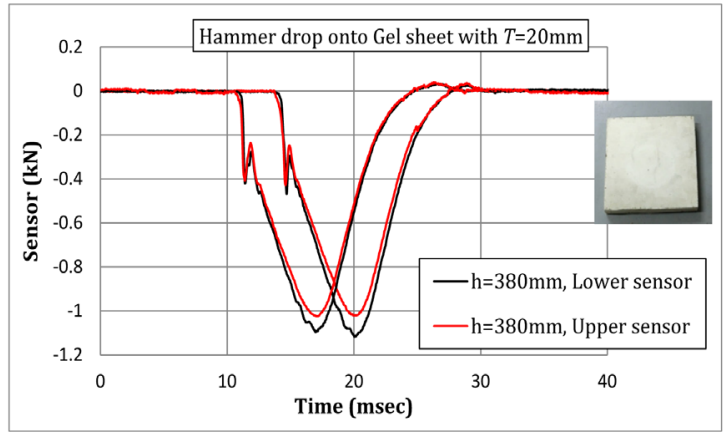

(a)

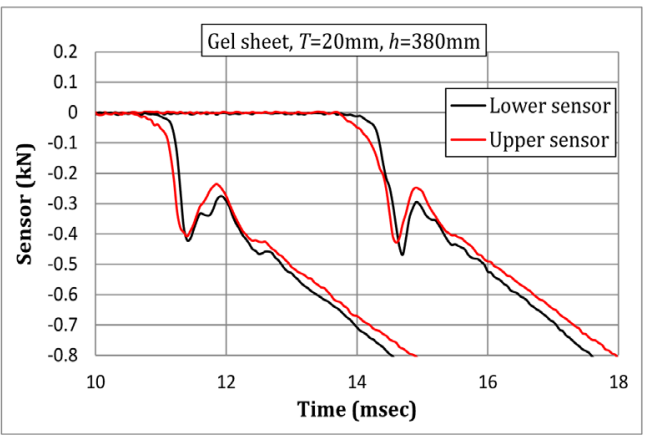

(b)

Figure 8. (a) Impact force waveform induced by the hammer drop onto a gel sheet with a 20 mm thickness; (b) Enlarged figure of the thorn part. 


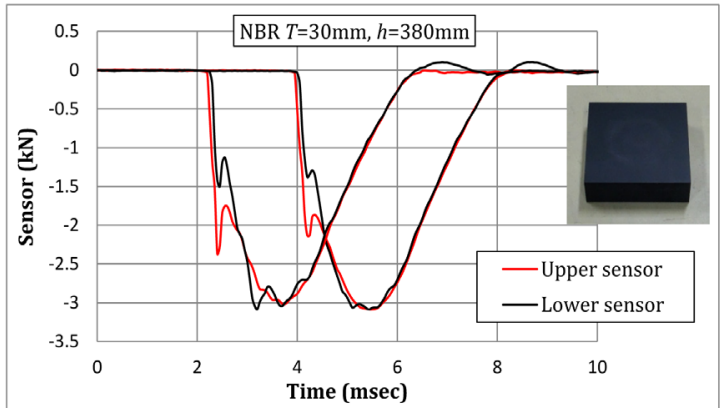

(a)

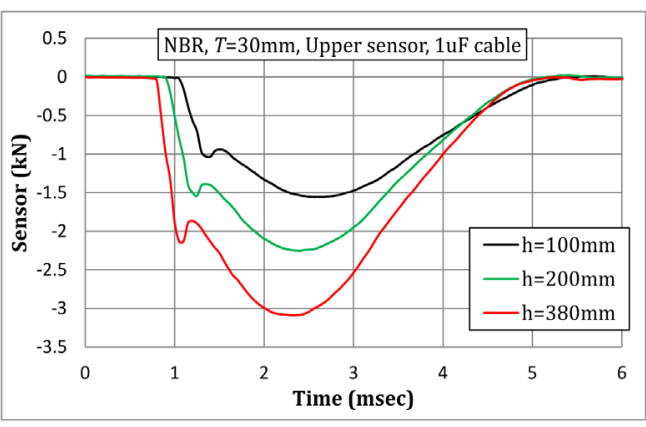

(b)

Figure 9. Impulsive force induced by the hammer drop onto NBR with $T=30 \mathrm{~mm}$.

$h=380 \mathrm{~mm}\left(\dot{\varepsilon}_{0}=91 \mathrm{sec}^{-1}\right)$. The upper sensor output shows a relatively large thorn, and the lower sensor output shows a smaller thorn. The slopes $\theta$ of the upper and lower sensor outputs are almost the same. Figure 9(b) compares the impact force waveforms of the upper sensor for drop heights of $h=100,200$, and $380 \mathrm{~mm}$. In the figure, when the impact velocity $V_{0}$ is faster, the slope $\theta$ of the rising segment and the height of the thorn peak are larger. This means that the slope $\theta$ and the height of the thorn peak are dependent on the impact velocity or strain rate.

\subsection{Impact Force of Oil Clay Plate under Flat Frontal Impact (Type II Waveform)}

Figure 10 shows the impact force waveform of an oil clay plate with a $50 \mathrm{~mm}$ thickness. The drop height is $h=$ 100 and $530 \mathrm{~mm}$. A large thorn is seen by the upper sensor, and a small thorn is seen by the lower sensor. As the drop height increases ( $V_{0}$ becomes faster), the slope $\theta$ becomes larger, and the thorn peak becomes taller. The thorns are more obvious than those of other materials. From the enlarged view, the thorn shape is close to an isosceles triangle.

\subsection{Impact Force of Low-Rebound Urethane Foam under Flat Frontal Impact (Type II Waveform)}

Figure 11 shows the impact force waveform of low-rebound urethane foam with a $40 \mathrm{~mm}$ thickness. The drop height is $h=380 \mathrm{~mm}$. In this case, the bottom diameter of the drop hammer is 60 and $75 \mathrm{~mm}$. In the figure, the steep slope of the rising segment is bent approximately 90 degrees at a certain force level. Also, a small thorn can be seen at the bent corner. Since low-rebound urethane foam is a high-viscosity material, the material shows a strong viscous resistance (hardening) until reaching a certain compressive stress.

\subsection{Impact Force of Ham and Edible Meat under Flat Frontal Impact (Type II Waveform)}

Figure 12 shows the impact force waveforms of sliced ham $(T=21 \mathrm{~mm})$, pork ham steak $(T=19 \mathrm{~mm})$, pork $(T$ $=25 \mathrm{~mm})$, and konjac $(T=20 \mathrm{~mm})$. The drop heights are all $h=380 \mathrm{~mm}$. The solid curve represents the upper sensor output, and the dashed curve represents the lower sensor output. We can see the steep slope $\theta$ in the ascending segment and the thorns in both the upper and lower sensor outputs.

Table 1 compares the falling momentum of the drop hammer and the impulse of the impact force waveform. The impulse is calculated by numerically integrating the area of the waveform. The impulse is about $10 \%$ larger than the falling momentum.

\section{Measurement of Triple-Strike Waveform}

Thin aluminum circular plates with a diameter of $25 \mathrm{~mm}$ and different thicknesses of $d=0.5,1.0,2.0,3.0$, and $5.0 \mathrm{~mm}$ are prepared. Each plate is attached to the bottom of the drop hammer with adhesive tape, as shown in Figure 13(a). Then, the hammer is dropped from $h=380 \mathrm{~mm}$ onto the NBR plate with a $30 \mathrm{~mm}$ thickness. The hammer bottom collides in two stages. The contact area at the first impact is a circle with a $25 \mathrm{~mm}$ diameter, and the second is a ring-shape area with a diameter range from 25 to $60 \mathrm{~mm}$. 


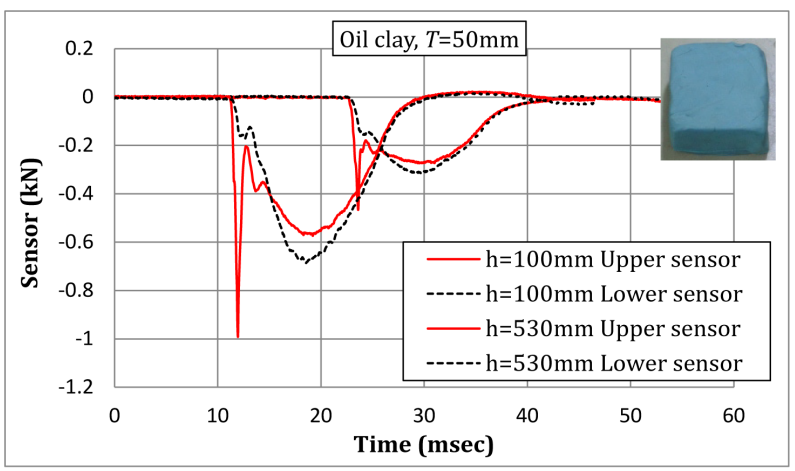

Figure 10. Impact force induced by the hammer drop onto oil clay with a $50 \mathrm{~mm}$ thickness.

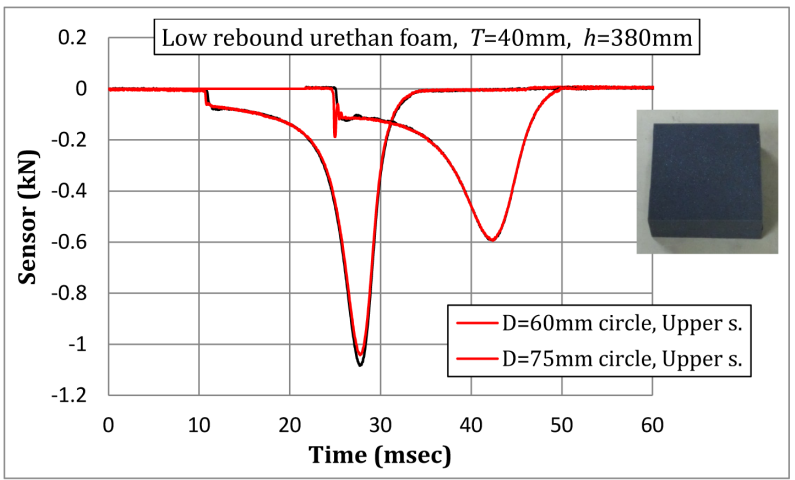

Figure 11. Impact force induced by the hammer drop onto low-rebound urethane foam.

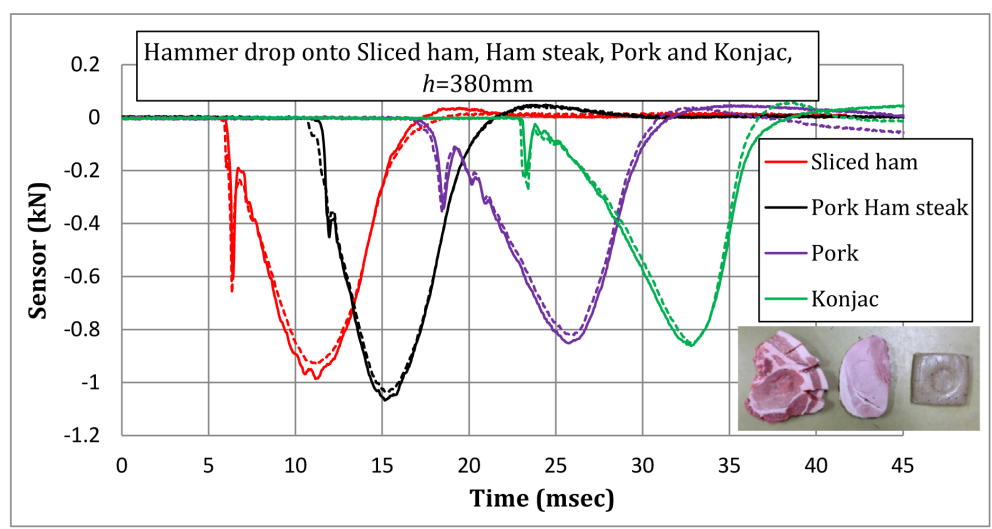

Figure 12. Impact force induced by the hammer drop onto sliced ham, pork ham steak, pork and konjac.

Table 1. Momentum and impulse of impact force induced by the hammer drop.

\begin{tabular}{|c|c|c|c|c|c|}
\hline \multirow{2}{*}{$\begin{array}{l}\text { Mass of drop weight } \\
\qquad m(\mathrm{~kg})\end{array}$} & \multirow{2}{*}{$\begin{array}{c}\text { Falling height } \\
h(\mathrm{~m})\end{array}$} & \multirow{2}{*}{$\begin{array}{l}\text { Momentum } \\
m V(\mathrm{~N} \cdot \mathrm{sec})\end{array}$} & \multirow{2}{*}{ Material } & \multirow{2}{*}{$\begin{array}{c}\text { Thickness } \\
T(\mathrm{~mm})\end{array}$} & Impulse $(\mathrm{N} \cdot \mathrm{sec})$ \\
\hline & & & & & Lower sensor \\
\hline \multirow{4}{*}{1.9} & \multirow{4}{*}{0.38} & \multirow{4}{*}{5.19} & Sliced ham & 21 & 5.92 \\
\hline & & & Pork ham steak & 19 & 5.58 \\
\hline & & & Pork & 25 & 5.58 \\
\hline & & & Konjac & 20 & 5.28 \\
\hline
\end{tabular}




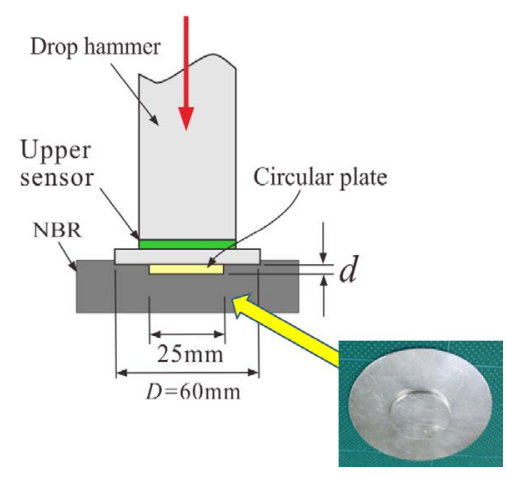

(a)

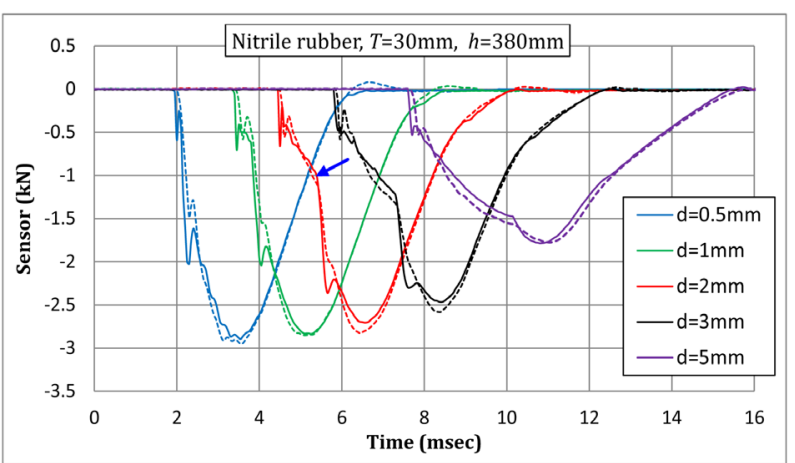

(b)

Figure 13. Measurement of triple-strike waveform of impact force. (a) Aluminum circular plate is attached to the bottom of the drop hammer; (b) Impact force induced by the hammer drop onto NBR with a $30 \mathrm{~mm}$ thickness.

Figure 13(b) shows the impact waveforms for all of the cases. The solid curves correspond to the upper sensor, and the dashed curves correspond to the lower sensor. We can see two thorn-shape waveforms and one mountain-shape waveform when $d$ is $3 \mathrm{~mm}$ or less. The first thorn is due to the impact by the small circular plate. The second thorn is due to the impact by the ring-shape area. The bending point between the first and second thorns indicates the start of contact of the ring-shape area (shown by the blue arrow in the case of $d=2 \mathrm{~mm}$ ).

In the typical double-strike phenomenon, the second strike makes a mountain-shape waveform. Therefore, the authors think that the second thorn such as that shown in Figure 13(b) will occur only if the drop hammer strikes the virgin surface of the soft material.

\section{Mechanism of Thorn-Shape Waveform and Double-Strike Phenomenon}

\subsection{Contact Time of Thorn-Shape Waveform}

Table 2 shows the contact time $t_{c}$ of the thorn-shape waveform (approximated by an isosceles triangle) and $\dot{\varepsilon}_{0}$ for some soft materials. The value of $t_{c}$ ranges between $0.4 \mathrm{msec}$ and $1.0 \mathrm{msec}$. Figure 14 shows the impact force waveform of a thick aluminum plate punched by a plastic hand hammer (PVC head). Its $t_{c}$ is $0.74 \mathrm{msec}$, and this value is close to those of soft materials. The material hardness is the main factor that governs $t_{c}$. Therefore, this result means that the soft materials reached almost the same hardness as the plastic hammer head at the thorn.

\subsection{Thickness Effect of Soft Material}

Figure 15 shows the impact force waveforms of oil clay plates with $T=10,20$, and $50 \mathrm{~mm}$. The solid curve is the upper sensor output, and the dashed curve is the lower sensor output. The drop heights are all $h=380 \mathrm{~mm}$ (impact velocity is $V_{0}=2.73 \mathrm{~m} / \mathrm{sec}$ ). The strain rate at contact is $\dot{\varepsilon}_{0}=V_{0} / T=273 \mathrm{sec}^{-1}, 137 \mathrm{sec}^{-1}$, and 54.6 $\sec ^{-1}$, respectively. In the figure, it is clear that the slope $\theta$ and the peak force of the thorn are influenced by the strain rate. The second mountain-shape waveform is also influenced by the strain rate.

The authors also performed experiments on cork sheet, sponge sheet, sponge rubber, paper clay, tofu, relatively smooth areas of human skin, and some hollow balls. Among them, all of the materials except the cork sheet showed a thorn-shape waveform.

\subsection{Mechanism of Thorn-Shape Waveform}

Figure 16 shows the author's understanding of the occurrence mechanism for the thorn-shape waveform based on the above experimental observations.

1) In the rising segment $P_{1}-P_{2}$ of Figure 16, the viscosity resistance is excessively large, and the waveform shows a steep slope $\theta$.

2) At the thorn peak $\mathrm{P}_{2}$, the viscosity resistance changes suddenly (viscosity transient). The large viscous resistance is broken by the thorn peak force and is softened to a certain extent. 
Table 2. Contact time of thorn-shape waveform for soft materials.

\begin{tabular}{ccc}
\hline Soft Material & $\begin{array}{c}\text { Contact Time } \\
t_{c}(\mathrm{msec})\end{array}$ & $\begin{array}{c}\mathrm{d} \varepsilon_{0} / \mathrm{d} t=V_{0} / T \\
\left(\mathrm{sec}^{-1}\right)\end{array}$ \\
\hline Ager Jelly & 0.5 & 91 \\
Gel Sheet & 0.8 & 136 \\
Konjac & 0.8 & 68 \\
Oil Clay & 0.4 & 273 \\
Oil Clay & 1.0 & 136 \\
NBR & 0.5 & 91 \\
Low-Rebound Urethane Foam & 0.7 & 68 \\
\hline
\end{tabular}

$h=380 \mathrm{~mm}$.
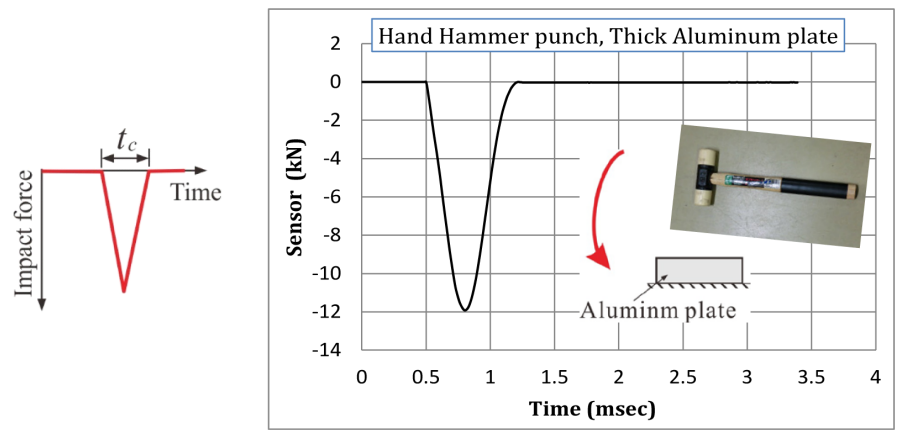

Figure 14. Impact force of aluminum plate punched by plastic hand hammer.

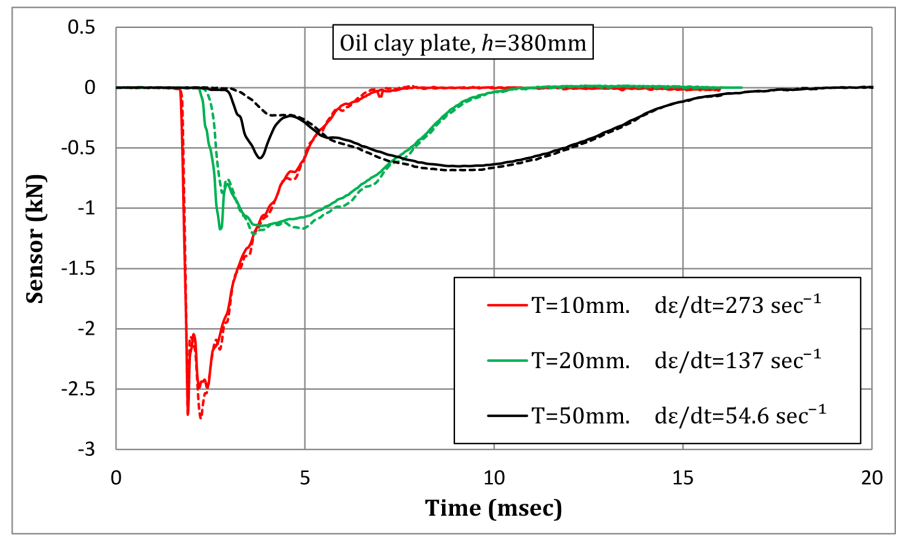

Figure 15. Thickness effect of oil clay plate on the impact force waveform. Drop heights are all $h=380 \mathrm{~mm}$. Solid curve is upper sensor, and dashed curve is lower sensor.

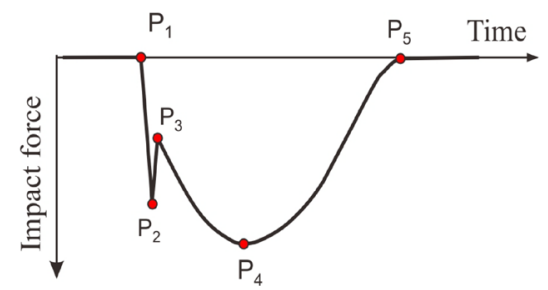

Figure 16. Mechanism of impact force waveform for soft materials based on the experimental observations. 


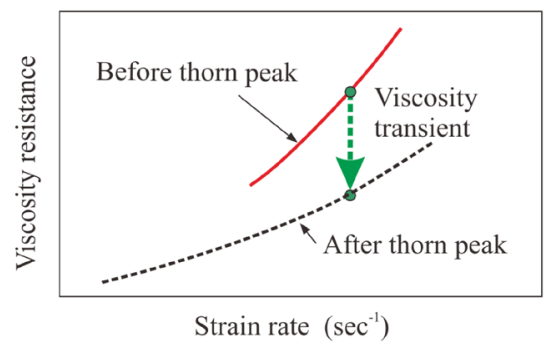

Figure 17. Viscosity transient vs. strain rate relationship.

3) When the viscosity resistance becomes small, the soft material is relaxed for a moment, and unloading occurs in the region $\mathrm{P}_{2}-\mathrm{P}_{3}$. When the softened material starts compressive deformation, the relative downward velocity between the hammer and the soft material surface decreases for a moment. This is the cause of unloading.

4) However, the hammer speed catches up to the soft material surface at the point $P_{3}$ and strikes it again. The second mountain-shape waveform shows a gentler slope $\alpha$ in the region $\mathrm{P}_{3}-\mathrm{P}_{4}$ because the viscosity resistance becomes small. The soft material shows a small viscosity resistance in the region $\mathrm{P}_{3}-\mathrm{P}_{5}$.

Based on the understanding of the above, the authors showed that the impact force waveform consisting of a thorn and mountain shape waveform can be simulated by using a simple dynamics model (SLS model) [7].

Figure 17 shows a schematic expression of the relationship between the viscosity resistance and the strain rate. The authors think that two types of viscosity resistance appear in the impact force of soft materials. One is the excessively large viscosity resistance (red curve) that appears in the first stage. The other is the small viscosity resistance (black dashed curve) that appears in the second stage. This viscosity transient (green dashed arrow) occurs at the thorn peak.

In some colloidal suspensions, the viscosity transient is known as "viscosity discontinuity" [8] [9]. Our experimental results indicate that the viscosity discontinuity may also occur in most soft materials.

It is known that the stress-strain curve of some soft materials under high strain rate is not a monotonically increasing function. It shows stress relaxation after the elastic peak [10]. This relaxation may be related to the viscosity discontinuity.

In running sports, it is known that a thorn-shape waveform called an "initial spike" can be observed [11]. When the heel strikes the ground first, it rebounds rapidly because the heel is hard. As a result, the heel generates a thorn-like waveform. Along with the movement of the center of gravity of the human body, the forefoot striking generates the second mountain-shape waveform. This is also understood as the double-strike phenomenon consisting of the first hard heel strike and the second soft forefoot strike.

\section{Conclusions}

Using compact drop test equipment, this study experimentally observed the impact force waveforms for several kinds of soft materials. The results obtained here are as follows:

1) Thorn-shape waveform of soft material appears clearly under the condition of a flat frontal impact. If either surface of the drop hammer or the soft material is curved, the thorn-shape waveform becomes unclear.

2) Most of the soft materials (except cork sheet) such as sponge, sponge rubber, nitrile rubber, oil clay, paper clay, low-rebound urethane foam, ham, pork, and konjac show a thorn-shape waveform under a flat frontal impact.

3) The thorn-shape waveform is close to an isosceles triangle. The rising slope $\theta$ and the height of the thorn peak are related to the strain rate dependence.

4) The thorn-shape waveform and succeeding mountain-shape waveform are induced by the double-strike phenomenon.

5) Viscosity discontinuity before and after the thorn waveform is thought to be the cause of the impact force waveform in soft materials.

\section{References}

[1] Zhao, H., Gary, G. and Klepaczko, J.P. (1997) On the Use of a Viscoelastic Split Hopkinson Pressure Bar. Internation- 
al Journal of Impact Engineering, 19, 319-330. http://dx.doi.org/10.1016/S0734-743X(96)00038-3

[2] Song, B., Chen, W.W., Ge, Y. and Weerasooriya, T. (2007) Radial Inertia Effects in Kolsky Bar Testing of Extra-Soft Specimens. Experimental Mechanics, 67, 659-670. http://dx.doi.org/10.1007/s11340-006-9017-5

[3] Kwon, J. and Subhash, G. (2010) Compressive Strain Rate Sensitivity of Ballistic Gelatin. Journal of Biomechanics, 43, 420-425. http://dx.doi.org/10.1016/j.jbiomech.2009.10.008

[4] Fujimoto, Y., Shintaku, E., Tanaka, Y. and Fujiyoshi, J. (2012) Pad Type Compressive Force Sensor Suitable for HighSpeed Impact Force Measurement. Transactions of the Japan Society of Mechanical Engineers, Series C, 78, 24382449. (in Japanese) http://dx.doi.org/10.1299/kikaic.78.2438

[5] Fujimoto, Y., Liu, C., Uesugi, T., Tanaka, Y. and Shintaku, E. (2013) Pipe Surface Sensor for Impulsive Force Measurement. Transactions of the Japan Society of Mechanical Engineers, Series C, 79, 113-123. (In Japanese) http://dx.doi.org/10.1299/kikaic.79.1949

[6] Fujimoto, Y., Liu, C., Tanaka, Y. and Shintaku, E. (2013) Measurement of Double-Strike Phenomenon of Impulsive Force Using Fluctuating Load Detection Plate. The Japanese Society for Experimental Mechanics, 13, 112-120. (In Japanese)

[7] Liu, C., Tanaka, Y. and Fujimoto, Y. (2015) Viscosity Transient Phenomenon during Drop Impact Testing and Its Simple Dynamics Model. World Journal of Mechanics, 5, 33-41. http://dx.doi.org/10.4236/wjm.2015.53004

[8] Hoffman, R.L. (1972) Discontinuous and Dilatant Viscosity Behavior in Concentrated Suspensions. I. Observation of a Flow Instability. Transactions of the Society of Rheology, 16, 155-173. http://dx.doi.org/10.1122/1.549250

[9] Bender, J. and Wagner, N.J. (1996) Reversible Shear Thickening in Monodisperse and Bidisperse Colloidal Dispersions. Journal of Rheology, 40, 889-915. http://dx.doi.org/10.1122/1.550767

[10] Richeton, J., Ahzi, S., Vecchio, K.S., Jiang, F.C. and Adharapurapu, R.R. (2006) Influence of Temperature and Strain Rate on the Mechanical Behavior of Three Amorphous Polymers: Characterization and Modeling of the Compressive Yield Stress. International Journal of Solids and Structures, 43, 2318-2335. http://dx.doi.org/10.1016/j.ijsolstr.2005.06.040

[11] Lieberman, D.E., Venkadesan, M., Werbel, W.A., Daoud, A.I., D’Andrea, S., Davis, I.S., Mang'eni, R.O. and Pitsiladis, Y. (2010) Foot Strike Patterns and Collision Forces in Habitually Barefoot Versus Shod Runners. Nature, 463, 531-535. http://dx.doi.org/10.1038/nature08723 